\title{
Patient Perspective on the Value of Genetic Counselling for Familial Pancreas Cancer
}

\author{
Jennifer E. Axilbund ${ }^{1,2}$, Kieran A. Brune ${ }^{3}$, Marcia I. Canto ${ }^{4}$, Brenda C. Brehon 5 , Lori D. Wroblewski ${ }^{5}$, Constance A. Griffin ${ }^{1-3}$ \\ 1Department of Oncology, School of Medicine, The Johns Hopkins University, Baltimore, Maryland, USA; 2Mid-Atlantic Cancer Genetics Network, The Johns \\ Hopkins University, Baltimore, Maryland, USA; ${ }^{3}$ Department of Pathology, School of Medicine, The Johns Hopkins University, Baltimore, Maryland, USA; \\ ${ }^{4}$ Department of Medicine, School of Medicine, The Johns Hopkins University, Baltimore, Maryland, USA; ${ }^{5}$ Department of Medicine, The Johns Hopkins Hospital, \\ Baltimore, Maryland, USA
}

Key words: pancreas cancer, genetic counselling

Corresponding author: Jennifer E. Axilbund, M.S., C.G.C., The Johns Hopkins Hospital, 500 North Broadway, Suite 410, Baltimore, Maryland 21205, USA, phone (410) 614-0378, fax (410) 955-4040, e-mail: axilbund@jhmi.edu

Submitted: 20 August 2005

Accepted: 28 August 2005

\begin{abstract}
Purpose: To assess patient views regarding the value of genetic counselling for familial pancreas cancer in the absence of predictive genetic testing.

Patients and methods: At-risk adults with three or more relatives with pancreas cancer received genetic counselling prior to research screening via endoscopic ultrasound. Questionnaires were mailed after the visit to assess perceived value of the counselling session.

Results: Ninety-three percent of respondents felt genetic counselling for pancreas cancer was helpful despite the lack of a causative gene, while only $7 \%$ felt that it should not be offered until such a gene is discovered. Over half of respondents believed the pancreas cancer in their family was caused by a gene mutation, and $42 \%$ thought they had inherited the mutation. The average perceived lifetime risk of developing pancreas cancer was $51 \%$, and $87 \%$ of respondents would ultimately seek predictive genetic testing. When more information is gained, $89 \%$ would be interested in another genetic counselling session, and $82 \%$ would recommend current genetic counselling for pancreas cancer to a friend or relative with a family history of the disease.

Conclusion: Despite the lack of an identified major causative gene for pancreas cancer, respondents found genetic counselling for this malignancy to be helpful. These patients perceive their personal cancer risk to be high, and would seek predictive genetic testing if it were available. Referral for genetic counselling should be offered to appropriate individuals.
\end{abstract}

\section{Introduction}

In its early days, genetic counselling was primarily offered to diagnose individuals with multiple congenital anomalies and to assess reproductive risk. With the discovery of genetic causes for various diseases, the scope of genetic counselling has broadened to include adult-onset disorders such as cancer. One challenge of genetic counselling is to recognize the specific syndrome affecting a particular individual, which can be accomplished either clinically through the recognition of physical features or constellation of diseases, or molecularly through the analysis of genes or proteins. Once a diagnosis is made, various health-related risks to the patient and his or her family members may be identified. Additionally, the pattern 
of inheritance is usually known, thereby allowing the genetic counsellor to provide recurrence estimates. Such estimates allow patients to make informed choices regarding health care and reproduction. Arguably, genetic counsellors are better able to facilitate patient decision-making when a definitive diagnosis can be made. In the absence of definitive information, however, the genetic counsellor still has the task of assisting the patient to make decisions and adjust to the disorder despite the uncertainty.

The discovery of the BRCA1 and BRCA2 genes in the 1990's has allowed health care providers to identify the "cause" of breast cancer in a subset of patients. For individuals affected with breast cancer, genetic testing for these two genes can be used to estimate the likelihood of future primary cancers, thereby potentially influencing medical management. Predictive testing is also available to unaffected family members, distinguishing those that require intensive screening or consideration of risk-reduction measures from those that are at general population risk for cancer and do not require excess care. Most health care providers view such delineation as a way of maximising the benefits of various medical services.

Numerous studies have assessed the impact of genetic counselling for breast cancer from the perspective of the at-risk patient, including effect on risk perception, anxiety and psychological affect. Three meta-analyses demonstrated that genetic counselling for hereditary breast cancer increases accuracy of risk perception and decreases generalized anxiety without an adverse impact on affective outcomes [1-3]. Although most analyses have focused on genetic counselling, genetic testing for BRCA1 and BRCA2 was clinically available at the time of study. Therefore, patients presumably had the opportunity to pursue predictive testing. Furthermore, genetics providers were able to provide numerical cancer risk estimates to patients using models developed by Claus and Gail $[4,5]$. The Claus model predicts breast cancer risk based on affected first and second-degree relatives and their respective ages at diagnosis. The Gail model, while only including affected first-degree relatives and not distinguishing between early and late-onset, also incorporates patient age, race, reproductive history and breast biopsy history. The advantage of these models is the ability to provide patients with personalised risk estimates.

Questions surround the utility of genetic counselling for diseases for which the causative genes have not yet been identified, such as pancreas cancer. As much as
$10 \%$ of pancreas cancer may be hereditary, yet there is no currently available genetic testing and lifetime risk estimates cannot be accurately provided in the overwhelming majority of cases. One wonders whether these at-risk patients have the same responses to genetic counselling as individuals who receive more personalised estimates and have the option of potentially informative genetic testing. Furthermore, it is unknown whether they find genetic counselling to be meaningful in the absence of such personalised information.

Klein [6] showed evidence of a rare major gene influencing risk for pancreas cancer; Klein [7] was also able to calculate standardised incidence ratios for individuals who have one, two or three first-degree relatives affected with pancreas cancer. Although these data may be used to provide personalised risk assessment to a small subset of familial pancreas cancer families, no data (such as the Claus or Gail models) currently exist to offer risk estimates to individuals with a more diffuse family history. This calls into question the value of genetic counselling for pancreas cancer.

We sought to assess the value of genetic counselling for familial pancreas cancer by surveying at-risk individuals who had received such counselling. The goal was to determine participants' views on the utility of genetic counselling in the absence of an identified major causative gene, interest in predictive genetic testing once it becomes available, and cancer risk perception based on family history.

\section{Patients and methods}

Adults with no personal history of pancreas cancer, but with three or more biological relatives with pancreas cancer (of whom at least one was a first-degree relative) were eligible to participate in a pancreas cancer screening study. Participants had to be at least 40 years old or no more than 10 years younger than the youngest diagnosis of pancreas cancer in their family. Screening entailed computed tomography imaging (CT) and endoscopic ultrasound (EUS), and, if indicated, endoscopic retrograde cholangiopancreatography (ERCP). The entire protocol is outlined by Canto [8].

Genetic counselling was provided to all participants prior to invasive screening. All participants were counselled by one genetic counsellor (JA), usually with one cancer genetics physician in attendance (CG). Subjects received a letter following the visit which summarised information discussed at the counselling session. The content of the counselling session included 
a review of the family history that was previously collected and confirmed by the National Familial Pancreas Tumor Registry (NFPTR), a description of features suggestive of a hereditary cancer syndrome and a general explanation of autosomal dominant inheritance. Each pedigree was then examined for characteristics of five hereditary syndromes known to be associated with an increased risk for pancreas cancer: (1) hereditary breast and ovarian cancer (BRCA2), (2) familial atypical multiple mole and melanoma syndrome (FAMMM), (3) hereditary non-polyposis colorectal cancer (HNPCC), (4) PeutzJeghers syndrome (PJS) and (5) hereditary pancreatitis. The general features of each syndrome were discussed with the patient, and suggestive family history, or lack thereof, was emphasized. Genetic testing is commercially available for each of these syndromes, but was not provided as part of this study; however, participants were informed if their family history was suggestive of any of the above-named syndromes, and were offered a clinical appointment with our service to pursue testing.

The study population contained two groups with a total " $n$ " of 69. Group 1 (seen September 2000 through June 2001) consisted of participants counselled prior to the publication of data indicating the presence of BRCA2 mutations in families with pancreas cancer in the absence of breast and/or ovarian cancer. Group 2 (March 2002 through February 2004) was seen after the publication of these data; thus, their counselling differed slightly. Evaluation of the counselling process was conceived after completion of counselling for group 1, so this group was analysed separately to account for differences in counselling and time to follow-up.

There were 20 counsellees in group 1. Each was contacted by telephone to determine his or her willingness to complete a short, written questionnaire regarding the counselling session. Fifteen agreed to participate while five did not respond to our initial telephone message. Questionnaires were only mailed to those 15 who actively agreed to participate, making the " $n$ " for group 1 fifteen. The average time elapsed between counselling and mailing of the questionnaire was approximately one year. Fifty-four group 2 participants were asked at the conclusion of counselling if they wished to complete the questionnaire. All agreed to participate, making the " $\mathrm{n}$ " for group 2 fifty-four. The average time elapsed between counselling and mailing of the questionnaire was approximately one month.

Questionnaire responses were anonymous and returned in postage-paid envelopes. Six demographic questions included gender, age, race, marital status, education and annual household income. Eighteen evaluative statements were based on a 5-point Likert scale, ranging from "strongly agree" to "strongly disagree" (see Appendix). Four open-ended questions ascertained perceived lifetime risk of pancreas cancer, the most and least useful aspects of the genetic counselling session and any additional comments. Estimated time to complete the questionnaire was 10 minutes.

Quantitative data were analysed using Microsoft Access and Excel. Statistical analysis was performed with Chi-square tables. Qualitative data, such as free text comments, were analysed thematically by inspection.

This study was approved through the Johns Hopkins Medicine Institutional Review Board and Office of Human Subjects Research.

\section{Results}

Forty-five completed questionnaires were received out of 69 sent, for a response rate of $65 \%$. There was no difference in response rate between groups 1 and 2 or males and females. Educational background was not collected prior to the questionnaire, so response rate based on education level could not be computed. Characteristics of the respondents are presented in Table 1, and include the average number of relatives with pancreas cancer per respondent. Twenty-one of the respondents also had a family history of breast and/or ovarian cancer. Among them, two were suspicious for hereditary breast and ovarian cancer, while none were strongly suggestive of FAMMM, HNPCC, PJS or hereditary pancreatitis.

Six questions ascertained participant views regarding genetic counselling for familial pancreas cancer. Overall responses were favourable, indicating that respondents found the session to be beneficial. Groups 1 and 2 were

Table 1. Characteristics of 45 respondents

\begin{tabular}{ll}
\hline Characteristic & Percent \\
\hline gender & male (44\%); female (56\%) \\
\hline marital status & married (82\%) \\
\hline education & college (18\%); graduate school (58\%) \\
\hline average age & 56 years (range: $39-81)$ \\
\hline average \# PC per family & 3.68 (range: 3-7) \\
\hline$P C=$ pancreas cancer &
\end{tabular}




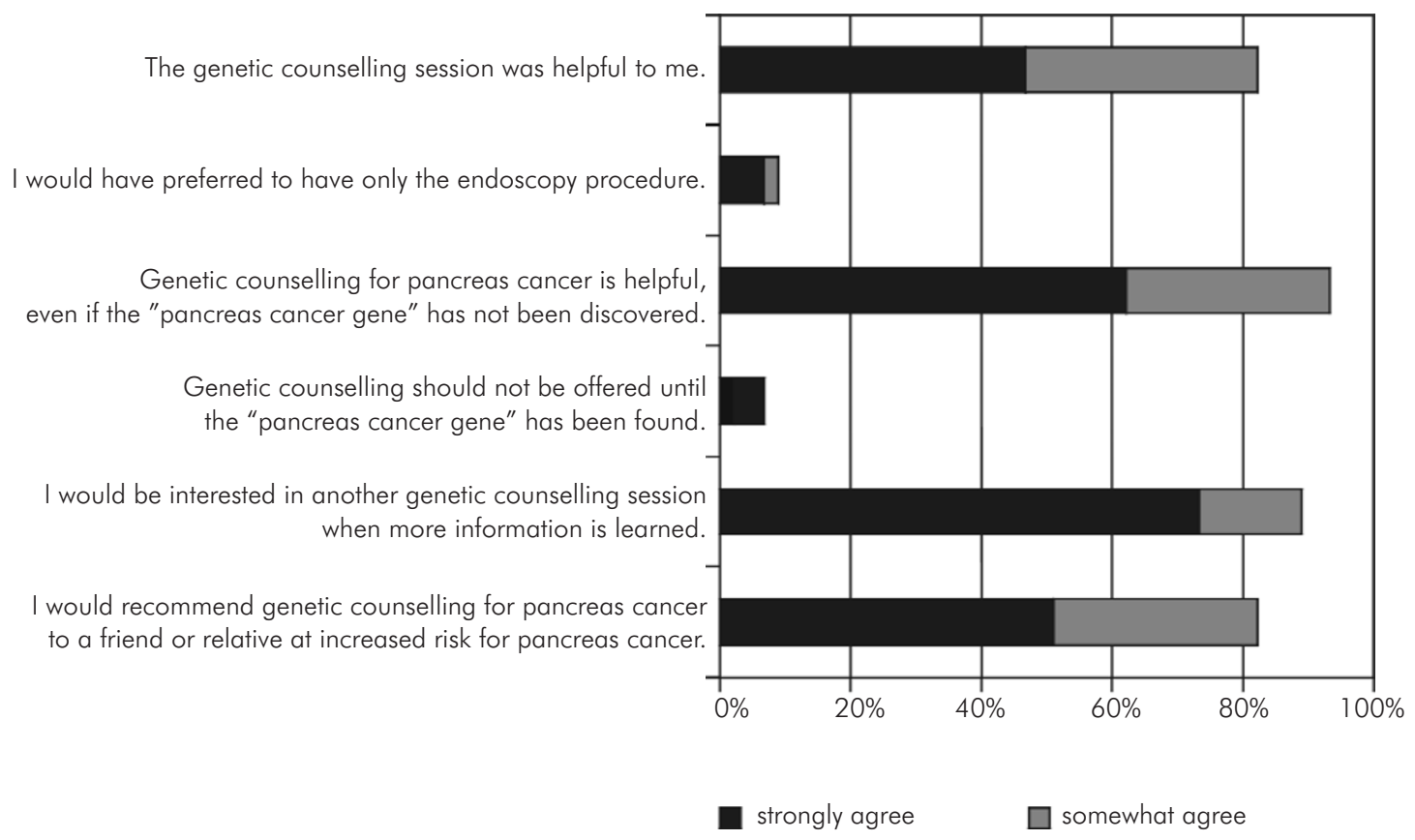

Fig. 1. Patient views on genetic counselling for familial pancreas cancer

If the "pancreas cancer gene" were found, I would want to be tested for it.

I do not feel that current genetic testing is likely to help me.

Even though the "pancreas cancer gene" has not been found, I want to get genetic testing for one or more of the syndromes discussed during genetic counselling.

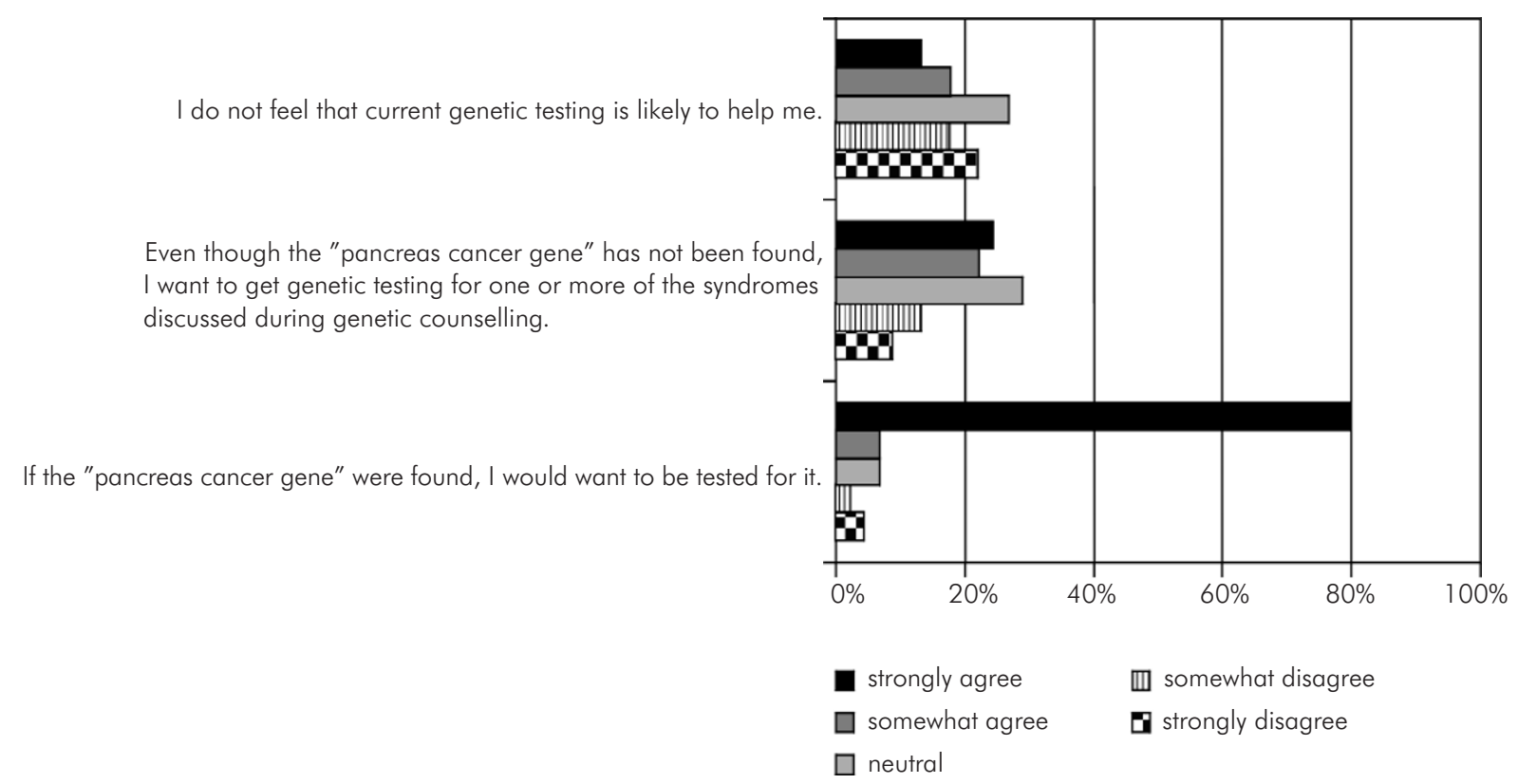

Fig. 2. Patient views on genetic testing for familial pancreas cancer

very similar, but group 1 respondents were more likely to prefer only the endoscopy procedure and not genetic counselling $(p \leq 0.025)$ and were less likely to find genetic counselling helpful in the absence of an identified "pancreas cancer gene" ( $p \leq 0.05)$. There were no significant differences between males and females or varying educational levels. Combined responses are presented in Figure 1.

Three questions ascertained participant view regarding genetic testing for familial pancreas cancer. Respondents were neutral on the ability of 
I think I inherited a gene mutation that causes pancreas cancer.

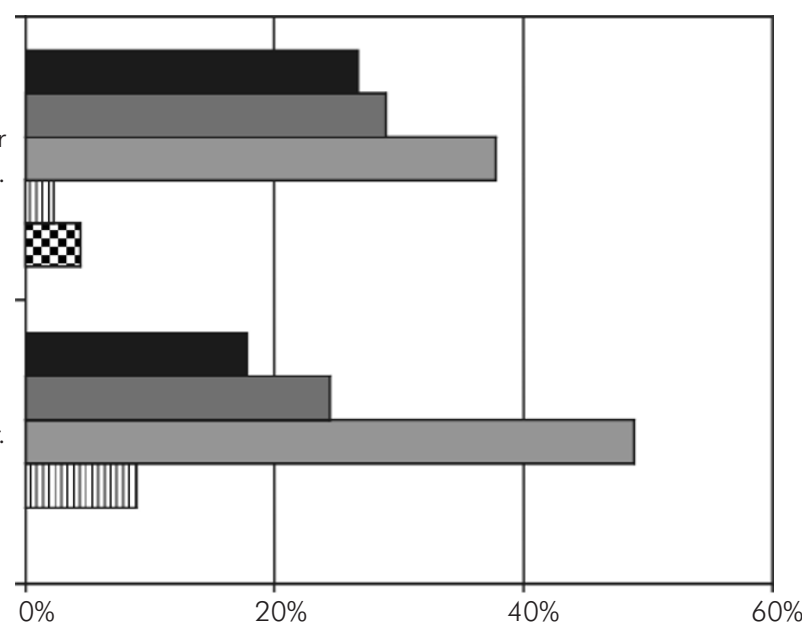

I think that the pancreas cancer in my family is caused by a gene mutation.

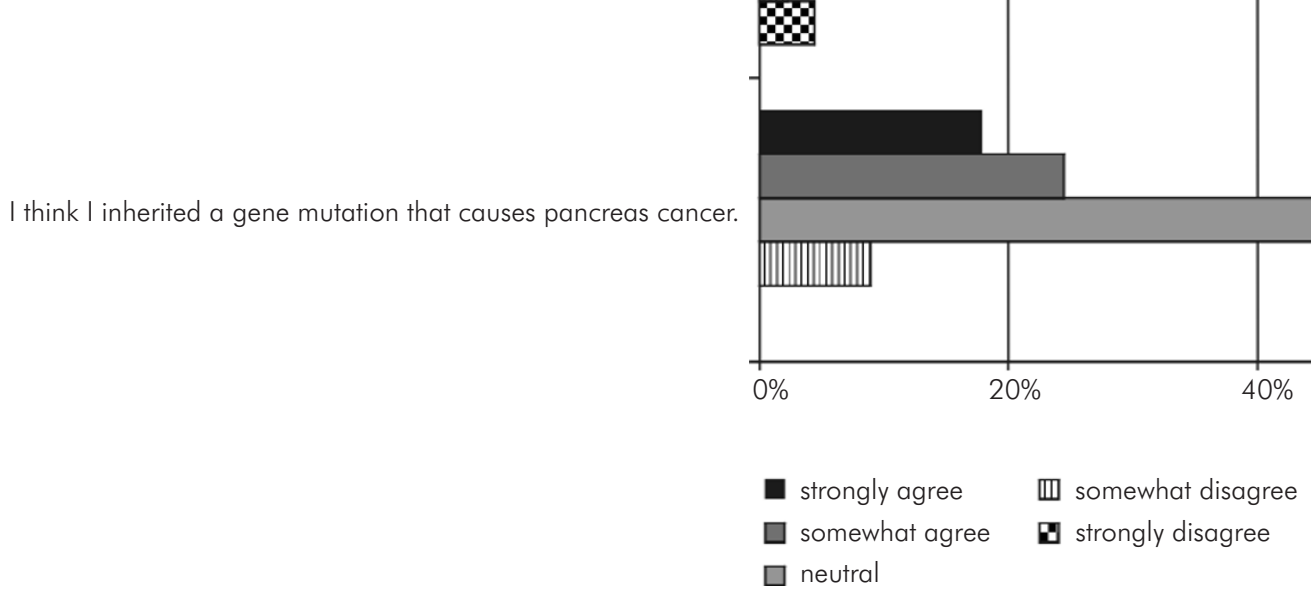

Fig. 3. Patient perception that family history is due to an inherited gene mutation

currently available testing to help them, but would want to be tested once the "pancreas cancer gene" is discovered. There were no significant differences between groups 1 and 2 or varying educational levels. Males and females were similar, though females were less inclined to pursue currently available genetic testing $(p \leq 0.05)$. Combined responses are presented in Figure 2.

Two questions ascertained participant perception of their risk of developing pancreas cancer, and respondents generally felt that they were at increased risk for this malignancy. There were no significant differences between groups 1 and 2, males and females or differing educational levels on these two evaluative questions; however, responses to an open-ended question asking participants to quantify their risk perception resulted in a significant difference between males and females. The mean lifetime risk estimate for all respondents was 50.8\% (range $1 \%$ to $100 \%$ ), with a mode of $50 \%(n=13)$. Males estimated a $38 \%$ lifetime risk while females estimated a $59 \%$ lifetime risk $(p \leq 0.01)$. Combined responses are presented in Figure 3.

Respondents cited explanation of the inheritance process, understanding cancer risk and contribution to general knowledge as the most useful aspects of the genetic counselling sessions. The least useful aspects were the limitations in the current state of knowledge and inability to provide personalised risk estimates rather than empiric risks.

\section{Discussion}

Respondents were largely in favour of genetic counselling for familial pancreas cancer, as more than $80 \%$ felt that it was helpful and agreed that genetic counselling should continue to be offered despite the inability to identify a major causative gene for this malignancy. Most respondents desired another genetic counselling session when more information is learned, and cited the lack of current knowledge as their biggest frustration. Although several respondents desired more personalised cancer risk assessment, they reported satisfaction with a better understanding of inheritance patterns and the various hereditary syndromes associated with pancreas cancer.

From these responses, one can conclude that individuals at increased risk for pancreas cancer benefit from genetic counselling, and that it should be accessible to appropriate individuals. Oncologists and internists who care for patients at risk for pancreas cancer should offer them a referral for genetic counselling by a qualified health care provider. Giardiello demonstrated that patients receiving genetic counselling from a provider less familiar with hereditary cancer syndromes often received 
inadequate counselling and would have been given incorrectly interpreted genetic test results [9]. Thus, a consultation with a specialty genetics clinic is recommended. The National Society of Genetic Counselors, Inc. maintains a directory of genetic counsellors at http://www.nsgc.org. Another source is the Cancer Genetics Services Directory by the National Cancer Institute at http://www.cancer.gov/search/ geneticsservices/.

As indicated, there were few differences between groups 1 and 2. However, those participants seen an average of a year prior to completion of the questionnaire were more likely to prefer only the endoscopy procedure, and were less likely to find the counselling helpful in the absence of available genetic testing. The majority of these individuals have had a second endoscopy procedure since their initial visit, as the screening study strives to evaluate participants annually. Therefore, the combination of the time elapsed and additional participation may account for the variation in group responses to these two statements.

Respondents' views on predictive genetic testing for hereditary pancreas cancer varied more. Two-thirds of respondents were either neutral or felt that currently available genetic tests were unlikely to be of benefit to them; however, almost half of all respondents were interested in testing for one of the syndromes discussed. This indicates a desire to explore opportunities to refine risk, no matter how small the likelihood of success. The need to refine risk is further exemplified by the $87 \%$ of respondents desiring testing once the "pancreas cancer gene" is discovered.

The 5 -year survival rate for patients with pancreas cancer is $12 \%$ to $20 \%$, if resectable, and less than $5 \%$ if unresectable. The American Society of Clinical Oncology recommends that genetic testing be offered if the patient has a personal or family history suggestive of a hereditary cancer syndrome, a genetic test is available that can be adequately interpreted, and the results will impact diagnosis or medical management of the patient and/or relatives [10]. Given the lack of proven screening for pancreas cancer, as well as the morbidity associated with prophylactic pancreatectomy, one could argue that predictive genetic testing for this malignancy should not necessarily be offered upon the discovery of a major causative gene. Rather, it should coincide with the availability of a safe and effective method to reduce the risk of advanced disease. Despite informed consent prior to participating in this screening study, respondents may have felt falsely reassured that their participation had a high likelihood of preventing advanced disease, thus explaining their desire to undergo genetic testing. However, desire to undergo genetic testing does not always equate with follow through, as has been demonstrated in individuals at increased risk for Huntington's disease, a neurological disorder with a devastating outcome and no effective intervention. Prior to the discovery of the associated gene, $57-84 \%$ of individuals at risk for Huntington's disease expressed a desire for predictive testing, while only $5-24 \%$ actually underwent such testing once it became available [11]. Therefore, once a major causative gene is discovered for pancreas cancer, the desire for predictive testing is anticipated to decrease from the $87 \%$ reported here.

Regarding risk perception, only 3 of 45 (7\%) respondents felt that the pancreas cancer in their family was unlikely to have been caused by a gene mutation. By contrast, over half of respondents felt that their family history was caused by a gene mutation, while one-third remained neutral. These results are as expected, as this is a group of people who sought out screening, thereby indicating a higher anxiety level about developing pancreas cancer. Individuals who do not suspect a hereditary aetiology to their family history are arguably less likely to participate in a study that poses a financial burden and the potential for an adverse complication secondary to the procedure.

When asked if they thought they had inherited the predisposition to pancreas cancer, nearly half of all respondents were neutral, while only 4 of the 45 (9\%) respondents believed they had not inherited it. Of note, among these latter four, two had responded that the cancer in their family was unlikely to be due to a gene mutation, thereby maintaining a consistent opinion. There are two potential explanations for the large neutral response to this question. One possibility is that the participants understood the $50 \%$ risk associated with autosomal dominant inheritance. The second possibility is that the respondents were unwilling to "commit" to a response, maintaining hope that they actually had not inherited the predisposition despite fear that they had. Overall, the response to this question was somewhat surprising, as one would expect more respondents to suspect they were at increased risk based on their participation in unproven screening.

When asked to quantify risk perception, the mean lifetime estimate was approximately $50 \%$. This is much greater than a geneticist would presume; although there is evidence of a "pancreas cancer gene", the penetrance is thought to be significantly below 100\%. Therefore, 
participant risk perception may be due to incomplete understanding of autosomal dominant inheritance, or due to a more basic perception of chance, such as, "either I will, or will not, develop pancreas cancer". Regardless, these results are consistent with previous reports focusing on individuals at increased risk for breast or colon cancer. Such research demonstrated that individuals at all levels of risk for cancer overestimate their own cancer risk, and that risk perception is not greatly influenced by genetic education and counselling [12]. Furthermore, subjects' decisions regarding genetic testing were primarily influenced by their perceived risk rather than actual risk status [12], which may explain why $87 \%$ of our participants ultimately desire predictive genetic testing. Also of interest was the difference in perceived lifetime risk between males and females in our study, with females estimating risk to be almost 20\% higher than males. However, this is also consistent with previous research indicating that females, in general, perceive their cancer risk to be higher than males do $[13,14]$.

The findings presented here are preliminary, and as such have several limitations. First, the sample size is small. Pancreas cancer is considered to be rare, and only $10 \%$ of pancreas cancer is likely to be hereditary. Therefore, there is not a large population of at-risk individuals from which to draw. Second, participants were ascertained through the screening study, and did not specifically seek out genetic counselling. Although this has the advantage of potentially being a less biased group, it is unknown whether individuals seeking clinical genetic counselling (i.e. requiring health insurance or self-payment) would have similar views. Third, the responses may be biased toward participants who favoured genetic counselling for familial pancreas cancer. Perhaps those who did not respond did not find the session to be valuable. Fourth, the participants were predominantly very well-educated, upper-middle class Caucasians. This was an uncontrollable consequence of the endoscopy screening study, which required that interested individuals travel, at their own expense, to the lone research site. In addition, certain components of the procedure were not covered by study funds, requiring that the patient self-pay or involve health insurance, thereby potentially eliciting a significant financial contribution on the part of the participant. Finally, prospective participants also needed to learn of the study's existence in the first place, which was not highly advertised due to a large pool of interested families already enrolled in a research registry. Furthermore, awareness of the registry, itself, is often gained through familiarity with the Internet or other resources, as $55 \%$ of registry families are self-referred versus joining at the recommendation of a health care provider. As a result, it is unknown whether individuals of differing socioeconomic status would view genetic counselling for pancreas cancer as favourably as those in the study described.

To address these limitations, as well as measure the psychological impact of genetic counselling for pancreas cancer on at-risk family members, a multi-centre consortium has been established. By involving more institutions, screening and genetic counselling can be offered to individuals of varying backgrounds with two goals: one, to improve screening for this deadly disease, and two, to determine the value of genetic counselling to a more diverse population.

\section{Acknowledgments}

This research was supported in part by: 1) National Cancer Institute CA78148-05; 2) National Cancer Institute Specialized Programs in Research Excellence (SPORE) 2 P50 CA62924- 10.

\section{References}

1. Braithwaite D, Emery J, Walter F, Prevost AT and Sutton S. Psychological impact of genetic counselling for familial cancer: a systematic review and meta-analysis. J Natl Cancer Inst 2004; 96: 122-133.

2. Butow PN, Lobb EA, Meiser B, Barratt A and Tucker KM. Psychological outcomes and risk perception after fenetic testing and counselling in breast cancer: A systematic review. Med J Aust 2003; 178: 77-81.

3. Meiser B and Halliday JL. What is the impact of genetic counselling in women at increased risk of developing hereditary breast cancer? A meta-analytic review. Soc Sci Med 2002; 54: 1463-1470.

4. Claus EB, Risch N and Thompson WD. Autosomal dominant inheritance of early-onset breast cancer. Implications for risk prediction. Cancer 1994; 73: 643-651.

5. Gail MH, Brinton LA, Byar DP, Corle DK, Green SB, Schairer C and Mulvihill JJ. Projecting individualized probabilities of developing breast cancer for white females who are being examined annually. J Natl Cancer Inst 1989; 81: 1879-1886.

6. Klein AP, Brune KA, Petersen GM, Goggins M, Tersmette AC, Offerhaus GJ, Griffin C, Cameron JL, Yeo CJ, Kern S and Hruban RH. Prospective risk of pancreatic cancer in familial pancreatic cancer kindreds. Cancer Res 2004; 64: 2634-2638.

7. Klein AP, Beaty TH, Bailey-Wilson JE, Brune KA, Hruban RH and Petersen GM. Evidence for a major gene influencing risk of pancreatic cancer. Genet Epidemiol 2002; 23: 133-149.

8. Canto MI, Goggins M, Yeo CJ, Griffin C, Axilbund JE, Brune K, Ali SZ, Jagannath S, Petersen GM, Fishman EK, Piantadosi S, Giardiello FM and Hruban RH. Screening for pancreatic neoplasia in high-risk individuals: an EUS-based approach. Clin Gastroenterol Hepatol 2004; 2: 606-621.

9. Giardiello FM, Brensinger JD, Petersen GM, Luce MC, Hylind LM, Bacon JA, Booker SV, Parker RD and Hamilton SR. The use and interpretation of commercial APC gene testing for familial adenomatous polyposis. N Engl J Med 1997; 336: 823-827.

10. American Society of Clinical Oncology policy statement update: Genetic testing for cancer susceptibility. J Clin Oncol 2003; 21: 1-10. 
11. Creighton S, Almqvist EW, MacGregor D, Fernandez B, Hogg H, Beis J, Welch JP, Riddell C, Lokkesmoe R, Khalifa M, MacKenzie J, Sajoo A, Farrell S, Robert F, Shugar A, Summers A, Meschino W, Allingham-Hawkins D, Chiu T, Hunter A, Allanson J, Hare H, Schween J, Collins L, Sanders S, Greenberg C, Cardwell S, Lemire E, MacLeod P and Hayden MR. Predictive, pre-natal and diagnostic genetic testing for Huntington's disease: the experience in Canada from 1987 to 2000. Clin Genet 2003; 63: 462-475.

12. Croyle RT and Lerman C. Risk communication in genetic testing for cancer susceptibility. J Nat Cancer Inst Monogr 1999; 25: 59-66.

13. Honda K and Neugut Al. Associations between perceived cancer risk and established risk factors in a national community sample. Cancer Detect Prev 2004; 28: 1 -7.

14. Helzlsouer KJ, Ford DE, Hayward RS, Midzenski M and Perry H. Perceived risk of cancer and practice of cancer prevention behaviors among employees in an oncology center. Prev Med 1994; 23: 302-308.

\section{APPENDIX}

1) I knew beforehand that I would receive genetic counselling as part of my research visit.

2) Before genetic counselling, I had already read or heard a fair amount about hereditary pancreas cancer.

3) The genetic counselling session was helpful to me.

4) I would have preferred to have only the endoscopy procedure and not genetic counselling.

5) I would have preferred more information about hereditary pancreas cancer.

6) Scientists do not currently know enough about hereditary pancreas cancer to help me.

7) Even though the "pancreas cancer gene" has not been found, I still want to get genetic testing for one or more of the syndromes discussed during genetic counselling.

8) I do not feel that current genetic testing is likely to help me.

9) I would recommend genetic counselling for pancreas cancer to a friend or relative with a family history of pancreas cancer.

10) The genetic information was too complex.

11) The written summary of the visit was useful.

12) I will share the written summary with my family members.

13) I think genetic counselling for pancreas cancer is helpful, even if the "pancreas cancer gene" has not been found.

14) If the "pancreas cancer gene" were found, I would want to be tested for it.

15) I do not think genetic counselling should be offered for pancreas cancer until the "pancreas cancer gene" has been found.

16) I would be interested in another genetic counselling session when more information is learned about pancreas cancer.

17) I think that the pancreas cancer in my family is caused by a gene mutation.

18) I think I inherited a gene mutation that causes pancreas cancer. 